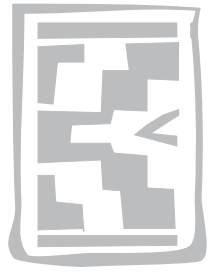

\title{
Ticks associated with the three largest wild ruminant species in southern Africa
}

I.G. HORAK ${ }^{1 *}$, H. GOLEZARDY² and A.C. UYS ${ }^{2}$

\begin{abstract}
HORAK, I.G., GOLEZARDY, H. \& UYS, A.C. 2007. Ticks associated with the three largest wild ruminant species in southern Africa. Onderstepoort Journal of Veterinary Research, 74:231-242

The objective of this study was to assess the host status of the three largest southern African wild ruminants, namely giraffes, Giraffa camelopardalis, African buffaloes, Syncerus caffer, and eland, Taurotragus oryx for ixodid ticks. To this end recently acquired unpublished data are added here to already published findings on the tick burdens of these animals, and the total numbers and species of ticks recorded on 12 giraffes, 18 buffaloes and 36 eland are summarized and discussed. Twenty-eight ixodid tick species were recovered. All stages of development of ten species, namely Amblyomma hebraeum, Rhipicephalus (Boophilus) decoloratus, Haemaphysalis silacea, Ixodes pilosus group, Margaropus winthemi, Rhipicephalus appendiculatus, Rhipicephalus evertsi evertsi, Rhipicephalus glabroscutatum, Rhipicephalus maculatus and Rhipicephalus muehlensi were collected. The adults of 13 species, of which the immature stages use small mammals as hosts, namely Haemaphysalis aciculifer, Hyalomma glabrum, Hyalomma marginatum rufipes, Hyalomma truncatum, Ixodes rubicundus, Rhipicephalus capensis, Rhipicephalus exophthalmos, Rhipicephalus follis, Rhipicephalus gertrudae, Rhipicephalus lounsburyi, Rhipicephalus lunulatus, Rhipicephalus pravus group and Rhipicephalus simus, were also collected.
\end{abstract}

Keywords: African buffaloes, eland, Giraffa camelopardalis, giraffes, ixodid ticks, Syncerus caffer, Taurotragus oryx, tick burdens

\section{INTRODUCTION}

Although numerous collections of ticks have been made from giraffes, Giraffa camelopardalis, African buffaloes, Syncerus caffer and eland, Taurotragus

* Author to whom correspondence is to be directed. E-mail: ivan.horak@up.ac.za

1 Department of Veterinary Tropical Diseases, Faculty of Veterinary Science, University of Pretoria, Onderstepoort, 0110 South Africa; Division of Parasitology, Onderstepoort Veterinary Institute, Onderstepoort, 0110 South Africa; and Department of Zoology and Entomology, University of the Free State, P.O. Box 339, Bloemfontein, 9300 South Africa

2 Department of Veterinary Tropical Diseases, Faculty of Veterinary Science, University of Pretoria, Onderstepoort, 0110 South Africa. A.C. Uys' present address: P.O. Box 652, Newlands, 0049 South Africa

Accepted for publication 23 March 2007-Editor oryx (Theiler 1962; Yeoman \& Walker 1967; Baker \& Keep 1970; Walker 1974; Norval 1983), it would seem that few attempts have been made to determine the total tick burdens of these large animals. This is perhaps not surprising considering the amount of assistance required to collect ticks from dead or immobilized animals of this size, and the time that has to be spent identifying and counting immature ticks in representative samples of these collections and often all the several thousand adult ticks that a single large herbivore may harbour. Amongst those who have attempted to do so are Horak, Potgieter, Walker, De Vos \& Boomker (1983), Rechav, Zeederberg \& Zeller (1987), Horak, Fourie, Novellie \& Williams (1991a), Horak, Anthonissen, Krecek \& Boomker (1992a) and Zieger, Horak, Cauldwell \& Uys (1998). Prior as well as subsequent to these published studies the opportunity to sam- 
ple more of these large herbivores has arisen and the present paper reports the results of the latter collections. However, since we thought it unlikely that, in the near future, similar exhaustive tick collections would be made from such large animals in southern Africa, we decided to combine the earlier published data with the more recent findings in order to give a more comprehensive overview of the numbers and species of ticks that may infest giraffes, African buffaloes and eland in a variety of habitats.

Giraffes occur in several associations of dry savanna, varying from scrub to woodland. They do not occur in forest or desert and generally are not found in open plains. They are present in north-eastern Limpopo and Mpumalanga Provinces, South Africa, and in the north-eastern parts of Namibia (Skinner \& Smithers 1990), but in recent times have been introduced into a number of regions in South Africa in which they previously did not occur.

African buffaloes prefer savanna-type habitats and need a plentiful supply of grass, shade and water. They occur in herds, which increase in size in the dry season, but become more fragmented during the wet season because of the usual plentiful supply of water and grazing (Skinner \& Smithers 1990). The current distribution of African buffaloes in South Africa is mostly patchy. Large numbers are present in the Kruger National Park (KNP) and the HluhluweiMfolozi Park in the north-eastern regions of the Limpopo, Mpumalanga and KwaZulu-Natal Provinces, with smaller populations in national, provincial and privately owned reserves in these and nearly all other provinces of South Africa.

Eland are gregarious animals usually occurring in small herds, and are as at home in arid semi-desert scrub associations as they are in montane grassland (Skinner \& Smithers 1990). Historically they were present virtually throughout South Africa, but overexploitation reduced their numbers. However, because they are a sought after species, reintroductions have resulted in their now occupying virtually the same regions in which they originally occurred.

One of the serious shortcomings of the past and current studies is that they are biased towards localities and seasons at which animals were, or have been, made available for survey purposes and are thus not necessarily representative of the entire ixodid tick fauna present within the geographic distribution of the host species being examined. Nevertheless a remarkably large number of tick species were recovered. Moreover, because of the large size of the hosts, large numbers of adult ticks were collected (Gallivan \& Horak 1997), and the greater certainty with which the adult ticks can be identified has augmented the accuracy of our identification of the immature stages, and hence our results.

\section{MATERIALS AND METHODS}

The localities at which animals were examined, including those of the earlier studies of Horak et al. (1983, 1991a, 1992a), Rechav et al. (1987) and Zieger et al. (1998), are summarized in Table 1. With the exception of Free State and Gauteng Provinces at least one of the three large wild ruminant species was sampled in each province of the Republic of South Africa. In addition, six of the giraffes were examined in the Etosha National Park in northern Namibia (Horak et al. 1992a), and two of the eland on a game ranch in Central Province, Zambia (Zieger et al. 1998).

Ticks were recovered from the animals in the earlier surveys as described by the authors of those studies. The carcasses of giraffes, African buffaloes and eland in the present surveys were processed for tick recovery and the ticks identified and counted as described by Horak, Boomker, Spickett \& De Vos (1992b). When animals had been immobilized, and not killed, an attempt was made to collect as many adult ticks as possible, with particular attention being paid to the preferred sites of attachment of the various species.

A total of 12 giraffes, 18 African buffaloes and 36 eland were examined, but in the tables devoted to each host species only the results for those animals sampled within a particular tick species' distribution range are given for that particular tick.

\section{RESULTS AND DISCUSSION}

The species and numbers of ticks collected from the giraffes, buffaloes and eland are summarized in Tables 2, 3 and 4, and the total numbers of male and female ticks recovered and, where it was possible to calculate this, the ratio of males to females for each species are summarized in Table 5.

A calculated total of 450709 ticks, belonging to 28 species and three subspecies, were collected from the 66 animals examined. Of these 57688 were adults, of which at least half were identified and counted. Although this may seem like a large number of ticks, probably three times as many would 
TABLE 1 Localities at which large wild ruminants were examined for ixodid ticks

\begin{tabular}{|c|c|c|c|}
\hline $\begin{array}{l}\text { Country and } \\
\text { province }\end{array}$ & Locality & Co-ordinates & $\begin{array}{l}\text { Vegetation type (Van der Merwe } \\
\text { 1983; White 1983; Acocks 1988) }\end{array}$ \\
\hline \multicolumn{4}{|l|}{ South Africa } \\
\hline Western Cape & West Coast National Park & $33^{\circ} 06^{\prime} \mathrm{S}, 17^{\circ} 59^{\prime} \mathrm{E}$ & $\begin{array}{l}\text { Strandveld and patches of } \\
\text { Coastal Fynbos }\end{array}$ \\
\hline Eastern Cape & $\begin{array}{l}\text { Thomas Baines Nature Reserve } \\
\text { Andries Vosloo Kudu Reserve } \\
\text { Mountain Zebra National Park }\end{array}$ & $\begin{array}{l}33^{\circ} 23^{\prime} \mathrm{S}, 26^{\circ} 28^{\prime} \mathrm{E} \\
33^{\circ} 07^{\prime} \mathrm{S}, 26^{\circ} 40^{\prime} \mathrm{E} \\
32^{\circ} 15^{\prime} \mathrm{S}, 25^{\circ} 27^{\prime} \mathrm{E}\end{array}$ & $\begin{array}{l}\text { False Macchia, Eastern Province } \\
\text { Thornveld and Valley Bushveld } \\
\text { Valley Bushveld } \\
\text { Karroid Merxmeullera Mountain } \\
\text { Veld, replaced by Karoo on the } \\
\text { higher slopes and Karroid Broken } \\
\text { Veld in the north }\end{array}$ \\
\hline Northern Cape & Kgalagadi Transfrontier Park & $\begin{array}{l}24^{\circ} 45^{\prime}-26^{\circ} 28^{\prime} \mathrm{S} \\
20^{\circ} 00^{\prime}-20^{\circ} 50^{\prime} \mathrm{E}\end{array}$ & $\begin{array}{l}\text { Lightly wooded grassland on dune } \\
\text { crests and grassland in depres- } \\
\text { sions between dunes }\end{array}$ \\
\hline KwaZulu-Natal & $\begin{array}{l}\text { Eastern Shores Park } \\
\text { iMfolozi Nature Reserve }\end{array}$ & $\begin{array}{l}28^{\circ} 08^{\prime} \mathrm{S}, 32^{\circ} 30^{\prime} \mathrm{E} \\
28^{\circ} 15^{\prime} \mathrm{S}, 31^{\circ} 57^{\prime} \mathrm{E}\end{array}$ & $\begin{array}{l}\text { Zululand Palm Veld, subdivision } \\
\text { of Coastal Thornveld and Coastal } \\
\text { communities } \\
\text { Zululand Thornveld and Lowveld }\end{array}$ \\
\hline North West & SA Lombaard Reserve & $27^{\circ} 35^{\prime} \mathrm{S}, 25^{\circ} 29^{\prime} \mathrm{E}$ & Dry Cymbopogon-Themeda veld \\
\hline Mpumalanga & $\begin{array}{l}\text { Pretoriuskop (KNP) } \\
\text { Lower Sabie (KNP) } \\
\text { Mtethomusha Nature Reserve }\end{array}$ & $\begin{array}{l}25^{\circ} 10^{\prime} \mathrm{S}, 31^{\circ} 16^{\prime} \mathrm{E} \\
25^{\circ} 07^{\prime} \mathrm{S}, 31^{\circ} 55^{\prime} \mathrm{E} \\
25^{\circ} 29^{\prime} \mathrm{S}, 31^{\circ} 17^{\prime} \mathrm{E}\end{array}$ & $\begin{array}{l}\text { Lowveld Sour Bushveld } \\
\text { Lowveld } \\
\text { Lowveld }\end{array}$ \\
\hline \multicolumn{4}{|l|}{ Namibia } \\
\hline & Okaukuejo, Etosha National Park & $19^{\circ} 11^{\prime} \mathrm{S}, 15^{\circ} 55^{\prime} \mathrm{E}$ & Mopane savanna \\
\hline \multicolumn{4}{|l|}{ Zambia } \\
\hline Central & Mtendere Game Ranch & $15^{\circ} 05^{\prime} \mathrm{S}, 28^{\circ} 16^{\prime} \mathrm{E}$ & Miombo Woodland \\
\hline
\end{tabular}

KNP = Kruger National Park

have been recovered had the digestion technique of Van Dyk \& McKenzie (1992) been used. However, the fine structures of tick larvae and nymphs are often damaged during the period of digestion required in the latter technique, and thus, although tick recovery may be more complete, it is achieved at the expense of accurate species determination.

Two of the 28 species recovered are one-host ticks, namely Margaropus winthemi and Rhipicephalus (Boophilus) decoloratus. Five are two-host ticks, these being Hyalomma glabrum, Hyalomma marginatum rufipes, Hyalomma truncatum, Rhipicephalus glabroscutatum and Rhipicephalus evertsi of which both subspecies of the latter, namely evertsi and mimeticus were collected. The remaining 21 species are all three-host ticks. All stages of development of Amblyomma hebraeum, R. (Boophilus) decoloratus,
Haemaphysalis silacea, Ixodes pilosus group, $M$. winthemi, Rhipicephalus appendiculatus, R. evertsi evertsi, R. glabroscutatum, Rhipicephalus maculatus and Rhipicephalus muehlensi were collected. Only the adults of 13 species, namely Haemaphysalis aciculifer, $H$. glabrum, $H$. marginatum rufipes, $H$. truncatum, Ixodes rubicundus, Rhipicephalus capensis, Rhipicephalus exophthalmos, Rhipicephalus follis, Rhipicephalus gertrudae, Rhipicephalus lounsburyi, Rhipicephalus lunulatus, Rhipicephalus pravus group and Rhipicephalus simus, of which the immature stages use small mammals as hosts, were recovered. In addition the larvae and nymphs of the South African tortoise tick, Amblyomma marmoreum, nymphs and adults of the tropical bont tick, Amblyomma variegatum, a single male Rhipicephalus longiceps, 30 Rhipicephalus supertritus males and 16 Rhipicephalus zambeziensis nymphs were re- 
Ticks associated with largest wild ruminant species in southern Africa

TABLE 2 Giraffes examined and the number infested within the distribution range of each tick

\begin{tabular}{|c|c|c|c|c|}
\hline Tick species & $\begin{array}{l}\text { Hosts } \\
\text { examined } \\
\text { (infested) }\end{array}$ & Life stage & $\begin{array}{l}\text { Total No. of } \\
\text { ticks }\end{array}$ & Locality \\
\hline Amblyomma hebraeum & $6(6)$ & $\begin{array}{l}\text { Larvae } \\
\text { Nymphs } \\
\text { Males } \\
\text { Females }\end{array}$ & $\begin{array}{r}773 \\
554 \\
2406 \\
512\end{array}$ & KNP \\
\hline Hyalomma marginatum rufipes & $12(8)$ & $\begin{array}{l}\text { Males } \\
\text { Females }\end{array}$ & $\begin{array}{r}374 \\
77\end{array}$ & KNP, Etosha \\
\hline Hyalomma truncatum & $12(12)$ & $\begin{array}{l}\text { Males } \\
\text { Females }\end{array}$ & $\begin{array}{r}1649 \\
619\end{array}$ & KNP, Etosha \\
\hline Rhipicephalus (Boophilus) decoloratus & $6(6)$ & $\begin{array}{l}\text { Larvae } \\
\text { Nymphs } \\
\text { Males } \\
\text { Females }\end{array}$ & $\begin{array}{r}2472 \\
2538 \\
1503 \\
821\end{array}$ & KNP \\
\hline Rhipicephalus appendiculatus & $6(6)$ & $\begin{array}{l}\text { Larvae } \\
\text { Nymphs } \\
\text { Males } \\
\text { Females }\end{array}$ & $\begin{array}{r}168 \\
682 \\
49 \\
4\end{array}$ & KNP \\
\hline Rhipicephalus evertsi evertsi & $6(6)$ & $\begin{array}{l}\text { Larvae } \\
\text { Nymphs } \\
\text { Males } \\
\text { Females }\end{array}$ & $\begin{array}{r}452 \\
190 \\
106 \\
20\end{array}$ & KNP \\
\hline Rhipicephalus evertsi mimeticus & $6(5)$ & $\begin{array}{l}\text { Males } \\
\text { Females }\end{array}$ & $\begin{array}{r}14 \\
5\end{array}$ & Etosha \\
\hline Rhipicephalus longiceps & $6(1)$ & Males & 1 & Etosha \\
\hline Rhipicephalus pravus group & $6(3)$ & $\begin{array}{l}\text { Males } \\
\text { Females }\end{array}$ & $\begin{array}{l}32 \\
20\end{array}$ & KNP \\
\hline Rhipicephalus simus & $6(4)$ & $\begin{array}{l}\text { Males } \\
\text { Females }\end{array}$ & $\begin{array}{r}22 \\
6\end{array}$ & KNP \\
\hline
\end{tabular}

KNP $=$ Kruger National Park

covered. Ten of the 28 tick species recovered occur only in South Africa. They are H. silacea, H. glabrum, I. pilosus, I. rubicundus, M. winthemi, R. capensis, $R$. follis, $R$. glabroscutatum and $R$. lounsburyi.

With the exception of ticks of the I. pilosus group and $I$. rubicundus, of which more females than males were recovered, considerably more males than females of all other species, of which both sexes were present, were collected. Mating in Ixodes species may occur in the preparasitic phase of the life cycle (Fourie \& Horak 1994), and many males thus never attach. This behaviour is probably largely responsible for the female-biased parasitic populations of the two ticks in this genus in the present study. The male-biased populations of the other tick species is due to the propensity of parasitic male ixodid ticks of several species to spend extended periods on their hosts (Londt 1976; Jordaan \& Baker 1981), whereas the females may require only a few days to two weeks to mate and engorge before detaching. Furthermore the larger size of engorging females is likely to make them more susceptible to removal by grooming or predation than the considerably smaller males.

\section{Amblyomma species}

The larvae of Amblyomma hebraeum infest a wide variety of hosts, including ungulates, carnivores, lagomorphs and birds, and all stages of development, but particularly the adults, infest very large hosts (Horak, Maclvor, Petney \& De Vos 1987a). The males may remain attached for several months (Jordaan \& Baker 1981), thus augmenting the already male-biased population of adult ticks. Ambly- 
TABLE 3 African buffaloes examined and the number infested within the distribution range of each tick

\begin{tabular}{|c|c|c|c|c|}
\hline Tick species & $\begin{array}{l}\text { Hosts } \\
\text { examined } \\
\text { (infested) }\end{array}$ & Life stage & $\begin{array}{l}\text { Total No. of } \\
\text { ticks }\end{array}$ & Locality \\
\hline Amblyomma hebraeum & $18(18)$ & $\begin{array}{l}\text { Larvae } \\
\text { Nymphs } \\
\text { Males } \\
\text { Females }\end{array}$ & $\begin{array}{r}47815 \\
6437 \\
6154 \\
1764\end{array}$ & $\begin{array}{l}\text { KNP, Eastern Shores, } \\
\text { iMfolozi, Thomas Baines, } \\
\text { Mtethomusha }\end{array}$ \\
\hline Amblyomma marmoreum & $18(4)$ & $\begin{array}{l}\text { Larvae } \\
\text { Nymphs }\end{array}$ & $\begin{array}{r}8 \\
26\end{array}$ & $\begin{array}{l}\text { KNP, Eastern Shores, } \\
\text { iMfolozi, Thomas Baines, } \\
\text { Mtethomusha }\end{array}$ \\
\hline Haemaphysalis silacea & $15(7)$ & $\begin{array}{l}\text { Larvae } \\
\text { Nymphs } \\
\text { Males } \\
\text { Females }\end{array}$ & $\begin{array}{r}81 \\
357 \\
135 \\
78\end{array}$ & $\begin{array}{l}\text { Eastern Shores, iMfolozi, } \\
\text { Thomas Baines }\end{array}$ \\
\hline Hyalomma truncatum & $17(2)$ & $\begin{array}{l}\text { Males } \\
\text { Females }\end{array}$ & $\begin{array}{l}6 \\
0\end{array}$ & $\begin{array}{l}\text { KNP, iMfolozi, } \\
\text { Mtethomusha }\end{array}$ \\
\hline Rhipicepalus (Boophilus) decoloratus & $18(11)$ & $\begin{array}{l}\text { Larvae } \\
\text { Nymphs } \\
\text { Males } \\
\text { Females }\end{array}$ & $\begin{array}{r}1640 \\
290 \\
248 \\
160\end{array}$ & $\begin{array}{l}\text { Eastern Shores, iMfolozi, } \\
\text { Thomas Baines, } \\
\text { Mtethomusha }\end{array}$ \\
\hline Rhipicephalus appendiculatus & $18(17)$ & $\begin{array}{l}\text { Larvae } \\
\text { Nymphs } \\
\text { Males } \\
\text { Females }\end{array}$ & $\begin{array}{r}139389 \\
32715 \\
1360 \\
774\end{array}$ & $\begin{array}{l}\text { KNP, Eastern Shores, } \\
\text { iMfolozi, Thomas Baines, } \\
\text { Mtethomusha }\end{array}$ \\
\hline Rhipicephalus evertsi evertsi & $18(15)$ & $\begin{array}{l}\text { Larvae } \\
\text { Nymphs } \\
\text { Males } \\
\text { Females }\end{array}$ & $\begin{array}{r}721 \\
35 \\
96 \\
45\end{array}$ & $\begin{array}{l}\text { KNP, Eastern Shores, } \\
\text { iMfolozi, Thomas Baines, } \\
\text { Mtethomusha }\end{array}$ \\
\hline Rhipicephalus follis & $1(1)$ & $\begin{array}{l}\text { Males } \\
\text { Females }\end{array}$ & $\begin{array}{l}40 \\
32\end{array}$ & Thomas Baines \\
\hline Rhipicephalus maculatus & $14(14)$ & $\begin{array}{l}\text { Larvae } \\
\text { Nymphs } \\
\text { Males } \\
\text { Females }\end{array}$ & $\begin{array}{r}15379 \\
16772 \\
946 \\
398\end{array}$ & Eastern Shores, iMfolozi \\
\hline Rhipicephalus muehlensi & $16(15)$ & $\begin{array}{l}\text { Larvae } \\
\text { Nymphs } \\
\text { Males } \\
\text { Females }\end{array}$ & $\begin{array}{r}11775 \\
495 \\
193 \\
141\end{array}$ & $\begin{array}{l}\text { Eastern Shores, iMfolozi, } \\
\text { Mtethomusha }\end{array}$ \\
\hline Rhipicephalus simus & $18(9)$ & $\begin{array}{l}\text { Males } \\
\text { Females }\end{array}$ & $\begin{array}{r}121 \\
64\end{array}$ & $\begin{array}{l}\text { KNP, Eastern Shores, } \\
\text { iMfolozi, Mtethomusha }\end{array}$ \\
\hline
\end{tabular}

KNP = Kruger National Park

omma hebraeum is present in the Bushveld and Lowveld regions of northern, eastern and southeastern South Africa (Walker \& Olwage 1987).

Amblyomma marmoreum is known as the South African tortoise tick, and all stages of development may be found on a variety of these reptiles, with leopard tortoises, Geochelone pardalis, generally the most heavily infested (Horak, McKay, Heyne \& Spickett 2006). The larvae infest a wide variety of mammals and birds, and are often found on mammals and birds of several species that are present within the tick's distribution range (Horak et al. 2006).

Amblyomma variegatum does not occur in South Africa, but is present in most sub-Saharan countries 
Ticks associated with largest wild ruminant species in southern Africa

TABLE 4 Eland examined and the number infested within the distribution range of each tick

\begin{tabular}{|c|c|c|c|c|}
\hline Tick species & $\begin{array}{l}\text { Hosts exam- } \\
\text { ined (infested) }\end{array}$ & Life stage & $\begin{array}{l}\text { Total No. of } \\
\text { ticks }\end{array}$ & Locality \\
\hline Amblyomma hebraeum & $4(4)$ & $\begin{array}{l}\text { Larvae } \\
\text { Nymphs } \\
\text { Males } \\
\text { Females }\end{array}$ & $\begin{array}{r}32394 \\
2405 \\
3488 \\
530\end{array}$ & $\begin{array}{l}\text { KNP, Andries Vosloo } \\
\text { Kudu Reserve, Thomas } \\
\text { Baines NR }\end{array}$ \\
\hline Amblyomma marmoreum & $15(8)$ & Larvae & 167 & $\begin{array}{l}\text { KNP, Mountain Zebra } \\
\text { NP, AV Kudu Reserve, } \\
\text { Thomas Baines NR }\end{array}$ \\
\hline Amblyomma variegatum & $2(2)$ & $\begin{array}{l}\text { Nymphs } \\
\text { Males } \\
\text { Females }\end{array}$ & $\begin{array}{r}4 \\
60 \\
26\end{array}$ & Mtendere Game Ranch \\
\hline Haemaphysalis aciculifer & $2(1)$ & Males & 2 & KNP \\
\hline Haemaphysalis silacea & $4(4)$ & $\begin{array}{l}\text { Larvae } \\
\text { Nymphs } \\
\text { Males } \\
\text { Females }\end{array}$ & $\begin{array}{r}7686 \\
933 \\
1409 \\
386\end{array}$ & $\begin{array}{l}\text { Andries Vosloo Kudu } \\
\text { Reserve, Thomas Baines } \\
\text { NR }\end{array}$ \\
\hline Hyalomma glabrum & $11(11)$ & $\begin{array}{l}\text { Males } \\
\text { Females }\end{array}$ & $\begin{array}{l}955 \\
191\end{array}$ & Mountain Zebra NP \\
\hline Hyalomma marginatum rufipes & $16(16)$ & $\begin{array}{l}\text { Males } \\
\text { Females }\end{array}$ & $\begin{array}{l}907 \\
470\end{array}$ & $\begin{array}{l}\text { Kgalagadi Transfrontier } \\
\text { Park, Thomas Baines } \\
\text { NR, SA Lombaard NR }\end{array}$ \\
\hline Hyalomma truncatum & $32(27)$ & $\begin{array}{l}\text { Males } \\
\text { Females }\end{array}$ & $\begin{array}{r}1702 \\
650\end{array}$ & $\begin{array}{l}\text { Mountain Zebra NP, } \\
\text { Kgalagadi Transfrontier } \\
\text { Park, West Coast NP, SA } \\
\text { Lombaard NR }\end{array}$ \\
\hline Ixodes pilosus group & $4(4)$ & $\begin{array}{l}\text { Larvae } \\
\text { Nymphs } \\
\text { Males } \\
\text { Females }\end{array}$ & $\begin{array}{r}2817 \\
177 \\
22 \\
64\end{array}$ & $\begin{array}{l}\text { Andries Vosloo Kudu } \\
\text { Reserve, Thomas Baines } \\
\text { NR }\end{array}$ \\
\hline Ixodes rubicundus & $11(7)$ & $\begin{array}{l}\text { Males } \\
\text { Females }\end{array}$ & $\begin{array}{l}34 \\
81\end{array}$ & Mountain Zebra NP \\
\hline Margaropus winthemi & $11(9)$ & $\begin{array}{l}\text { Larvae } \\
\text { Nymphs } \\
\text { Males } \\
\text { Females }\end{array}$ & $\begin{array}{r}12792 \\
6874 \\
1915 \\
893\end{array}$ & Mountain Zebra NP \\
\hline Rhipicephalus (Boophilus) decoloratus & $8(5)$ & $\begin{array}{l}\text { Larvae } \\
\text { Nymphs } \\
\text { Males } \\
\text { Females }\end{array}$ & $\begin{array}{r}3106 \\
3988 \\
3581 \\
848\end{array}$ & $\begin{array}{l}\text { KNP, AV Kudu Reserve, } \\
\text { Thomas Baines NR, } \\
\text { Mtendere Game Ranch }\end{array}$ \\
\hline Rhipicephalus appendiculatus & $8(8)$ & $\begin{array}{l}\text { Larvae } \\
\text { Nymphs } \\
\text { Males } \\
\text { Females }\end{array}$ & $\begin{array}{r}18025 \\
1489 \\
8106 \\
3345\end{array}$ & $\begin{array}{l}\text { KNP, AV Kudu Reserve, } \\
\text { Thomas Baines NR, } \\
\text { Mtendere Game Ranch }\end{array}$ \\
\hline Rhipicephalus capensis & $2(2)$ & $\begin{array}{l}\text { Males } \\
\text { Females }\end{array}$ & $\begin{array}{r}1506 \\
408\end{array}$ & West Coast NP \\
\hline Rhipicephalus evertsi evertsi & $34(31)$ & $\begin{array}{l}\text { Larvae } \\
\text { Nymphs } \\
\text { Males } \\
\text { Females }\end{array}$ & $\begin{array}{r}6900 \\
2128 \\
1574 \\
392\end{array}$ & $\begin{array}{l}\text { KNP, Mountain Zebra } \\
\text { NP, AV Kudu Reserve, } \\
\text { Thomas Baines NR, } \\
\text { West Coast NP, SA } \\
\text { Lombaard NR, Mtendere } \\
\text { Game Ranch }\end{array}$ \\
\hline
\end{tabular}


TABLE 4 Cont.

\begin{tabular}{|c|c|c|c|c|}
\hline Tick species & $\begin{array}{l}\text { Hosts exam- } \\
\text { ined (infested) }\end{array}$ & Life stage & $\begin{array}{l}\text { Total No. of } \\
\text { ticks }\end{array}$ & Locality \\
\hline Rhipicephalus exophthalmos & $11(1)$ & $\begin{array}{l}\text { Males } \\
\text { Females }\end{array}$ & $\begin{array}{l}0 \\
2\end{array}$ & Mountain Zebra NP \\
\hline Rhipicephalus follis & $11(11)$ & $\begin{array}{l}\text { Males } \\
\text { Females }\end{array}$ & $\begin{array}{l}983 \\
447\end{array}$ & $\begin{array}{l}\text { Mountain Zebra NP, } \\
\text { Thomas Baines NR }\end{array}$ \\
\hline Rhipicephalus gertrudae & $2(1)$ & $\begin{array}{l}\text { Males } \\
\text { Females }\end{array}$ & $\begin{array}{l}4 \\
2\end{array}$ & West Coast NP \\
\hline Rhipicephalus glabroscutatum & $14(13)$ & $\begin{array}{l}\text { Larvae } \\
\text { Nymphs } \\
\text { Males } \\
\text { Females }\end{array}$ & $\begin{array}{r}6510 \\
2846 \\
868 \\
499\end{array}$ & $\begin{array}{l}\text { Mountain Zebra NP, } \\
\text { Andries Vosloo Kudu } \\
\text { Reserve, West Coast NP }\end{array}$ \\
\hline Rhipicephalus lounsburyi & $11(2)$ & Males & 3 & Mountain Zebra NP \\
\hline Rhipicephalus Iunulatus & $2(2)$ & $\begin{array}{l}\text { Males } \\
\text { Females }\end{array}$ & $\begin{array}{r}218 \\
48\end{array}$ & Mtendere Game Ranch \\
\hline Rhipicephalus simus & $6(4)$ & $\begin{array}{l}\text { Males } \\
\text { Females }\end{array}$ & $\begin{array}{r}39 \\
5\end{array}$ & $\begin{array}{l}\text { KNP, AV Kudu Reserve, } \\
\text { Thomas Baines NR }\end{array}$ \\
\hline Rhipicephalus supertritus & $2(2)$ & Males & 30 & Mtendere Game Ranch \\
\hline Rhipicephalus zambeziensis & $2(1)$ & Nymphs & 16 & KNP \\
\hline
\end{tabular}

KNP $=$ Kruger National Park

$N P=$ National Park

$\mathrm{NR}=$ Nature Reserve

to the north (Walker \& Olwage 1987). Its host spectrum is similar to that of $A$. hebraeum and its presence on the two eland examined in Zambia is thus to be expected.

\section{Haemaphysalis species}

The two male Haemaphysalis aciculifer recovered from an eland in the KNP during September 1979 (Horak et al. 1983; Table 4), were the only specimens of this species reported in the park until 2000, when Horak, Braack, Fourie \& Walker (2000) recorded 13 males and two females from a honey badger, Mellivora capensis. This tick is seldom recovered in large numbers but appears to be present in north-eastern Mpumalanga Province and thence along the eastern and southern coastal and adjacent inland regions to the south-western region of the Cape Province (Theiler 1962; Horak, Keep, Spickett \& Boomker 1989; Horak \& Boomker 1998).

Haemaphysalis silacea is present in north-eastern KwaZulu-Natal and in the Eastern Cape Provinces (Walker 1991), and is associated with vegetation classified as Valley Bushveld (Acocks 1988) and with greater kudus, Tragelaphus strepsiceros, common duikers, Sylvicapra grimmia, Cape grysbok,
Rhaphicerus melanotis, and helmeted guineafowls, Numida meleagris (Horak \& Knight 1986; Maclvor \& Horak 2003). Seven of the 18 buffaloes and all four eland examined in Valley Bushveld-type vegetation in the north-eastern KwaZulu-Natal and the Eastern Cape Provinces were infested.

\section{Hyalomma species}

Delpy (1949) described the tick now known as Hyalomma glabrum as Hyalomma rufipes glabrum, and the latter name persisted until Theiler (1956) raised it to species level as Hyalomma glabrum. In the same year Hoogstraal (1956) synonymized it with Asian Hyalomma marginatum turanicum, and since then it has been considered identical to the latter tick. Recent studies have, however, revealed that it is a separate taxon, and Apanaskevich \& Horak (2006) have subsequently reinstated it as Hyalomma glabrum. Howell, Walker \& Nevill (1978) have illustrated its distribution (as H. m. turanicum) in the central and western regions of South Africa. The adults prefer large animals such as eland and Cape mountain zebras, Equus zebra zebra, and its immature stages infest scrub hares, Lepus saxatilis, and ground-frequenting birds (Apanaskevich \& Horak 2006). 
Ticks associated with largest wild ruminant species in southern Africa

TABLE 5 Male to female ratios of tick species collected from giraffes, African buffaloes and eland in southern Africa

\begin{tabular}{|c|c|c|c|c|}
\hline \multirow{2}{*}{ Tick species } & \multicolumn{2}{|c|}{ Total No. of adult ticks } & \multicolumn{2}{|c|}{ Male:female ratio } \\
\hline & Male & Female & Male & Female \\
\hline Amblyomma hebraeum & 12048 & 2806 & 4.29 & 1.0 \\
\hline Amblyomma variegatum & 60 & 26 & 2.31 & 1.0 \\
\hline Haemaphysalis aciculifer & 2 & 0 & - & - \\
\hline Haemaphysalis silacea & 1544 & 464 & 3.33 & 1.0 \\
\hline Hyalomma glabrum & 955 & 191 & 5.00 & 1.0 \\
\hline Hyalomma marginatum rufipes & 1281 & 547 & 2.34 & 1.0 \\
\hline Hyalomma truncatum & 3357 & 1269 & 2.65 & 1.0 \\
\hline Ixodes pilosus group & 22 & 64 & 0.34 & 1.0 \\
\hline Ixodes rubicundus & 34 & 81 & 0.42 & 1.0 \\
\hline Margaropus winthemi & 1915 & 893 & 2.14 & 1.0 \\
\hline Rhipicephalus (Boophilus) decoloratus & 5332 & 1829 & 2.92 & 1.0 \\
\hline Rhipicephalus appendiculatus & 9515 & 4123 & 2.31 & 1.0 \\
\hline Rhipicephalus capensis & 1506 & 408 & 3.69 & 1.0 \\
\hline Rhipicephalus evertsi evertsi & 1776 & 457 & 3.89 & 1.0 \\
\hline Rhipicephalus evertsi mimeticus & 14 & 5 & 2.80 & 1.0 \\
\hline Rhipicephalus exophthalmos & 0 & 2 & - & - \\
\hline Rhipicephalus follis & 1023 & 479 & 2.14 & 1.0 \\
\hline Rhipicephalus gertrudae & 4 & 2 & 2.00 & 1.0 \\
\hline Rhipicephalus glabroscutatum & 868 & 499 & 1.74 & 1.0 \\
\hline Rhipicephalus longiceps & 1 & 0 & - & - \\
\hline Rhipicephalus lounsburyi & 3 & 0 & - & - \\
\hline Rhipicephalus lunulatus & 218 & 48 & 4.54 & 1.0 \\
\hline Rhipicephalus maculatus & 946 & 398 & 2.38 & 1.0 \\
\hline Rhipicephalus muehlensi & 193 & 141 & 1.37 & 1.0 \\
\hline Rhipicephalus pravus group & 32 & 20 & 1.60 & 1.0 \\
\hline Rhipicephalus simus & 182 & 75 & 2.43 & 1.0 \\
\hline Rhipicephalus supertritus & 30 & 0 & - & - \\
\hline Total (excluding Ixodes spp.) & 42805 & 14682 & 2.92 & 1.0 \\
\hline
\end{tabular}

The distribution of Hyalomma marginatum rufipes is more extensive than that of $H$. glabrum, which it almost entirely overlaps (Howell et al. 1978). Its host preferences are the same as those of the latter tick (Walker 1991), but include giraffes and buffaloes, which are generally not present within the distribution range of $H$. glabrum (Tables 2 and 3 ).

With the exception of the eastern coastal regions and some adjacent inland areas and the southern coastal regions, Hyalomma truncatum is present virtually throughout the country (Howell et al. 1978). The adults are found on the same large hosts as the former two ticks, and its immature stages infest scrub hares and rodents (Walker 1991).

\section{Ixodes species}

Ticks of the Ixodes pilosus group are present in the southern coastal and adjacent regions of the Western and Eastern Cape Provinces and in southeastern KwaZulu-Natal Province, where all stages of development may be encountered on antelopes, caracals, Caracal caracal and domestic dogs (Horak, Jacot Guillarmod, Moolman \& De Vos 1987b; Horak et al. 1989; Horak \& Boomker 1998; Horak \& Matthee 2003).

The colloquial name given to Ixodes rubicundus is Karoo paralysis tick, because of the paralysis associated with infestation of small domestic livestock in the Karoo regions of the country (Howell et al. 1978; Fourie \& Horak 1994). The hosts of the adults are wild and domestic ruminants, caracals and domestic dogs (Horak et al. 1987b, 1991a; Fourie \& Horak 1993; Horak \& Matthee 2003). The immature stages infest Smith's red rock rabbits, Pronolagus rupestris, caracals and rock elephant shrews, Elephantulus myurus (Horak et al. 1991a; Fourie, Horak \& Woodall 2005).

\section{Margaropus winthemi}

This tick is commonly known as the winter horse tick, and its distribution in the cooler higher-lying regions of the country suggests that its original hosts 
were Cape mountain zebras, on which it still occurs in very large numbers during the winter months (Horak et al. 1991a). The burdens of the eland (Table 4), examined at the same time as zebras in the Mountain Zebra National Park, although large, did not nearly approach those of the latter animals.

\section{Rhipicephalus species}

Rhipicephalus (Boophilus) decoloratus. Using molecular analyses supported by an analysis of morphological characters Murrell, Campbell \& Barker (2000) and Beati \& Keirans (2001) decided that the genus Rhipicephalus is paraphyletic with respect to the genus Boophilus and consequently Murrell \& Barker (2003) proposed the use of the above nomenclature. We have chosen to follow their proposal, although many people prefer to retain Boophilus as a generic name. Giraffes and eland can generally be considered as good hosts of this tick, whereas African buffaloes appear to be resistant to infestation, with more than half of the adult ticks reported here coming from a single, approximately 6-month-old calf. Norval (1984) and Horak, Golezardy \& Uys (2006) have discussed the apparent resistance of African buffaloes to artificial and natural infestations with this tick, and it would seem that this resistance is acquired, rather than innate.

Adult Rhipicephalus appendiculatus are common parasites of African buffaloes, eland, greater kudus, male nyalas, Tragelaphus angasii, and domestic cattle in wooded savannas from south-eastern South Africa in the south to Kenya and Uganda in the north (Walker, Keirans \& Horak 2000). The immature stages are found on the same hosts as the adults, but also infest smaller antelopes and scrub hares (Walker et al. 2000). The eland included in the present study and more particularly those from Zambia, were heavily infested with adult ticks (Table 4). The buffaloes, which were examined mainly during the early winter months, harboured very large numbers of larvae (Table 3).

Rhipicephalus capensis, Rhipicephalus follis, Rhipicephalus gertrudae and Rhipicephalus simus have several morphological characteristics in common and also have similar life cycles during which the immature stages infest murid rodents and the adults the larger species of antelopes (Walker et al. 2000). Adult R. gertrudae also infest domestic dogs and primates, including humans (Brain \& Bohrmann 1992; Horak, Fourie, Heyne, Walker \& Needham 2002; Horak \& Matthee 2003), while those of $R$. simus infest domestic and wild equids, suids and carnivores (Horak et al. 1987b, 2000; Walker et al.
2000). Rhipicephalus capensis occurs almost exclusively in the western coastal regions of the Western Cape Province, South Africa; $R$. gertrudae is present in the same western regions, but its distribution extends east to the southern and southern-central regions of the country and north into Namibia; $R$. follis is only found in South Africa and is present to the east and north-east of $R$. gertrudae's distribution range and generally in mountainous terrain; while, with the exception of the more arid regions of central and western South Africa and southern Namibia, the distribution range of $R$. simus effectively overlays those of the other three species and extends north into Africa to a latitude of approximately $9^{\circ}$ south.

Rhipicephalus evertsi evertsi and Rhipicephalus evertsi mimeticus are two-host ticks and all stages of development may infest the same host species. Excluding the deserts and regions of high rainfall, the former tick is found throughout sub-Saharan Africa, whereas the latter is confined to the arid and semi-arid regions of Namibia, western Botswana and parts of Angola (Walker et al. 2000).

The adults of Rhipicephalus exophthalmos parasitize antelopes, domestic ruminants and scrub hares in the south-eastern and north-western regions of South Africa and in a broad, central band from the south to the north of Namibia (Walker et al. 2000). Its immature stages prefer elephant shrews, Elephantulus spp., as hosts (Fourie et al. 2005).

The two-host tick, Rhipicephalus glabroscutatum, is present in the fynbos and Karoo regions of the Western Cape Province, and also in the Karoo and the Valley Bushveld regions of the Eastern Cape Province (Walker et al. 2000). It is a common parasite of the feet and lower legs of small and large antelope in these regions (Horak \& Boomker 1998; Maclvor \& Horak 2003). Infestation with its adults is a contributory cause of foot abscess in domestic goats in the Valley Bushveld regions of the Eastern Cape Province (Maclvor \& Horak 1987).

The distribution in South Africa of Rhipicephalus maculatus and Rhipicephalus muehlensi is confined to the coastal bush and adjacent inland regions of north-eastern KwaZulu-Natal (Walker et al. 2000). The recovery of an $R$. maculatus nymph from the vegetation and adults from an elephant, Loxodonta africana, in the southern regions of the KNP (Braack, Maggs, Zeller \& Horak 1995), and of $R$. muehlensi adults from one of the buffaloes examined in the Mtethomusha Nature reserve, just south of the KNP, suggest that they have been introduced into these 
reserves on animals translocated from north-eastern KwaZulu-Natal. All stages of development of both ticks may infest the same host species, but the adults of $R$. maculatus prefer thick-skinned animals such as buffaloes and bush pigs, Potamochoerus larvatus, and those of $R$. muehlensi nyalas (Horak, Boomker \& Flamand 1991b; 1995).

Rhipicephalus longiceps is a rarely encountered tick and apparently is present only in certain regions of Namibia and Angola (Walker et al. 2000).

Rhipicephalus lounsburyi prefers the higher mountainous regions of the Eastern Cape Province (Walker et al. 2000) and two eland in the Mountain Zebra National Park were infested. The adults attach around the feet of their antelope and sheep hosts, while the only known hosts of its immature stages are four-striped grass mice, Rhabdomys pumilio, from which a larva and two nymphs have been collected (Walker et al. 2000; Horak, Fourie \& Braack 2005).

Although Rhipicephalus lunulatus is fairly widespread in sub-Saharan Africa its distribution in South Africa is limited to the eastern regions (Walker et al. 2000). The adults have a wide host range and would seem to attach around the feet and lower legs of their hosts (Walker et al. 2000). The eland examined in Zambia had fairly large burdens of adult ticks (Table 4), while a multimammate mouse, Mastomys sp., and a scrub hare examined on the same ranch as the eland were infested with nymphs (Zieger et al. 1998).

Ticks belonging to the Rhipicephalus pravus group have been collected from scrub hares in the KNP (Horak, Spickett, Braack \& Penzhorn 1993), and were assigned to Rhipicephalus sp. near pravus by Walker et al. (2000). However, the ticks collected in the same park from three of the giraffes, appear to us to be very similar, if not identical to the true $R$. pravus of East Africa.

The immature stages of Rhipicephalus supertritus are unknown, but are assumed to be similar in appearance to those of $R$. appendiculatus. It is apparently commonest in Central Africa, including northern Zimbabwe and Mozambique, parts of Zambia and southern Tanzania (Walker et al. 2000), and both eland examined in Zambia were infested.

\section{ACKNOWLEDGEMENTS}

We are most grateful to Ezemvelo KZN Wildlife, SANParks, and the Provincial Division of Nature
Conservation of the Eastern Cape Province for placing the animals included in the recent surveys at our disposal, and for providing assistance and facilities to process them for tick recovery. We are particularly indebted to Messrs Johan Sithole and Eddie Williams for their assistance in the latter respect. The University of Pretoria and the National Research Foundation provided funds for the conduct of this project. The publication of this work has been facilitated through the Integrated Consortium on Ticks and Tick-borne Diseases (ICTTD-3), financed by the International Cooperation Program of the European Union through Coordination Action Project No. 510561.

\section{REFERENCES}

ACOCKS, J.P.H. 1988. Veld types of South Africa with accompanying veld type map, $3^{\text {rd }}$ ed. (Memoirs of the Botanical Survey of South Africa, No. 57).

APANASKEVICH, D.A. \& HORAK, I.G. 2006. The genus Hyalomma Koch, 1844. I. Reinstatement of Hyalomma (Euhyalomma) glabrum Delpy, 1949 (Acari, Ixodidae) as a valid species with a re-description of the adults, the first description of the immature stages and notes on its biology. Onderstepoort Journal of Veterinary Research, 73:1-12.

BAKER, MAUREEN K. \& KEEP, M.E. 1970. Ckecklist of the ticks found on the larger game animals in the Natal game reserves. Lammergeyer, 12:41-47.

BEATI, L. \& KEIRANS, J.E. 2001. Analysis of the systematic relationships among ticks of the genera Rhipicephalus and Boophilus (Acari: Ixodidae) based on mitochondrial 12S ribosomal DNA gene sequences and morphological characters. Journal of Parasitology, 87:32-48.

BRAACK, L.E.O., MAGGS, K.A.R., ZELLER, D.A. \& HORAK, I.G. 1995. Exotic arthropods in the Kruger National Park, South Africa: modes of entry and population status. African Entomology, 3:39-48.

BRAIN, C. \& BOHRMANN, R. 1992. Tick infestation of baboons (Papio ursinus) in the Namib desert. Journal of Wildlife Diseases, 28:188-191.

DELPY, L.P. 1949. Révision par des voies expérimentales du genre Hyalomma C.L. Koch 1844 ( $2^{\mathrm{e}}$ partie). Annales de Parasitologie humaine et comparée, 24 (1-2):97-109.

FOURIE, L.J. \& HORAK, I.G. 1993. Sites of attachment of Karoo paralysis ticks (Ixodes rubicundus) on three cattle breeds. Journal of the South African Veterinary Association, 64:9091.

FOURIE, L.J. \& HORAK, I.G. 1994. The life cycle of Ixodes rubicundus (Acari: Ixodidae) and its adaptation to a hot, dry environment. Experimental and Applied Acarology, 18:23-35.

FOURIE, L.J., HORAK, I.G. \& WOODALL, P.F. 2005. Elephant shrews as hosts of immature ixodid ticks. Onderstepoort Journal of Veterinary Research, 72:293-301.

GALLIVAN, G.J. \& HORAK, I.G. 1997. Body size and habitat as determinants of tick infestations of wild ungulates in South Africa. South African Journal of Wildlife Research, 27:63-70.

HOOGSTRAAL, H. 1956. African Ixodoidea. I. Ticks of the Sudan (with special reference to Equatoria Province and with preliminary reviews of the genera Boophilus, Margaropus and Hyalomma). Washington DC: US Navy. 
HORAK, I.G., POTGIETER, F.T., WALKER, JANE B., DE VOS, V. \& BOOMKER, J. 1983. The ixodid tick burdens of various large ruminant species in South African nature reserves. Onderstepoort Journal of Veterinary Research, 50:221-228.

HORAK, I.G. \& KNIGHT, M.M. 1986. A comparison of the tick burdens of wild animals in a nature reserve and on an adjacent farm where tick control is practised. Journal of the South African Veterinary Association, 57:199-203.

HORAK, I.G., MACIVOR, K.M. DE F., PETNEY, T.N. \& DE VOS, V. 1987a. Some avian and mammalian hosts of Amblyomma hebraeum and Amblyomma marmoreum (Acari: Ixodidae). Onderstepoort Journal of Veterinary Research, 54:397-403.

HORAK, I.G., JACOT GUILLARMOD, AMY, MOOLMAN, L.C. \& DE VOS, V. 1987b. Parasites of domestic and wild animals in South Africa. XXII. Ixodid ticks on domestic dogs and on wild carnivores. Onderstepoort Journal of Veterinary Research, 54:573-580.

HORAK, I.G., KEEP, M.E., SPICKETT, A.M. \& BOOMKER, J. 1989. Parasites of domestic and wild animals in South Africa. XXIV. Arthropod parasites of bushbuck and common duiker in the Weza State Forest, Natal. Onderstepoort Journal of Veterinary Research, 56:63-66.

HORAK, I.G., FOURIE, L.J., NOVELLIE, P.A. \& WILLIAMS, E.J. 1991a. Parasites of domestic and wild animals in South Africa. XXVI. The mosaic of ixodid tick infestations on birds and mammals in the Mountain Zebra National Park. Onderstepoort Journal of Veterinary Research, 58:125-136.

HORAK, I.G., BOOMKER, J. \& FLAMAND, J.R.B. 1991b. Ixodid ticks and lice infesting red duikers and bushpigs in northeastern Natal. Onderstepoort Journal of Veterinary Research, 58:281-284.

HORAK, I.G., ANTHONISSEN, M., KRECEK, R.C. \& BOOMKER, J. 1992a. Arthropod parasites of springbok, gemsbok, kudus, giraffes and Burchell's and Hartmann's zebras in the Etosha and Hardap Nature Reserves, Namibia. Onderstepoort Journal of Veterinary Research, 59:253-257.

HORAK, I.G., BOOMKER, J., SPICKETT, A.M. \& DE VOS, V. 1992b. Parasites of domestic and wild animals in South Africa. XXX. Ectoparasites of kudus in the eastern Transvaal Lowveld and the eastern Cape Province. Onderstepoort Journal of Veterinary Research, 59:259-273.

HORAK, I.G., SPICKETT, A.M., BRAACK, L.E.O. \& PENZHORN, B.L. 1993. Parasites of domestic and wild animals in South Africa. XXXII. Ixodid ticks on scrub hares in the Transvaal. Onderstepoort Journal of Veterinary Research, 60:163-174.

HORAK, I.G., BOOMKER, J. \& FLAMAND, J.R.B. 1995. Parasites of domestic and wild animals in South Africa. XXXIV. Arthropod parasites of nyalas in north-eastern KwaZulu-Natal. Onderstepoort Journal of Veterinary Research, 62:171-179.

HORAK, I.G. \& BOOMKER, J. 1998. Parasites of domestic and wild animals in South Africa. XXXV. Ixodid ticks and bot fly larvae in the Bontebok National Park. Onderstepoort Journal of Veterinary Research, 65:205-211.

HORAK, I.G., BRAACK, L.E.O., FOURIE, L.J. \& WALKER, JANE B. 2000. Parasites of domestic and wild animals in South Africa. XXXVIII. Ixodid ticks collected from 23 wild carnivore species. Onderstepoort Journal of Veterinary Research, 67: 239-250.

HORAK, I.G., FOURIE, L.J., HEYNE, HELOISE, WALKER, JANE B. \& NEEDHAM, G.R. 2002. Ixodid ticks feeding on humans in South Africa: with notes on preferred hosts, geographic distribution, seasonal occurrence and transmission of pathogens. Experimental and Applied Acarology, 27:113136.
HORAK, I.G. \& MATTHEE, SONJA 2003. Parasites of domestic and wild animals in South Africa. XLIII. Ixodid ticks of domestic dogs and cats in the Western Cape Province. Onderstepoort Journal of Veterinary Research, 70:187-195.

HORAK, I.G., FOURIE, L.J. \& BRAACK, L.E.O. 2005. Small mammal hosts of immature ixodid ticks. Onderstepoort Journal of Veterinary Research, 72:255-261.

HORAK, I.G., MCKAY, I.J., HEYNE, HELOISE \& SPICKETT, A.M. 2006. Hosts, seasonality and geographic distribution of the South African tortoise tick, Amblyomma marmoreum. Onderstepoort Journal of Veterinary Research, 73:13-25.

HORAK, I.G., GOLEZARDY, H. \& UYS, A.C. 2006. The host status of African buffaloes, Syncerus caffer, for Rhipicephalus (Boophilus) decoloratus. Onderstepoort Journal of Veterinary Research, 73:193-198.

HOWELL, C.J., WALKER, JANE B. \& NEVILL, E.M. 1978. Ticks, mites and insects infesting domestic animals in South Africa. Part 1. Descriptions and biology. Pretoria: Department of Agricultural Technical Services, Republic of South Africa (Science Bulletin no. 393).

JORDAAN, J.O. \& BAKER, J.A.F. 1981. Survival rate on the host and mating capacity of Amblyomma hebraeum (Koch) male ticks, in Tick biology and control, edited by G.B. Whitehead \& J.D. Gibson. Tick Research Unit, Rhodes University, Grahamstown: $115-117$.

LONDT, J.G.H. 1976. Fertilization capacity of Boophilus decoloratus (Koch, 1844) (Acarina: Ixodidae). Onderstepoort Journal of Veterinary Research, 43:143-145.

MACIVOR, K.M. DE F. \& HORAK, I.G. 1987. Foot abscess in goats in relation to the seasonal abundance of adult Amblyomma hebraeum and adult Rhipicephalus glabroscutatum (Acari: Ixodidae). Journal of the South African Veterinary Association, 58:113-118.

MACIVOR, K.M. DE F. \& HORAK, I.G. 2003. Ixodid ticks of Angora and Boer goats, grysbok, common duikers, kudus and scrub hares in Valley Bushveld in the Eastern Cape Province. Onderstepoort Journal of Veterinary Research, 70:113120.

MURRELL, A., CAMPBELL, N.J.H. \& BARKER, S.C. 2000. Phylogenetic analysis of the rhipicephaline ticks indicates that the genus Rhipicephalus is paraphyletic. Molecular Phylogenetics and Evolution, 16:1-7.

MURRELL, A. \& BARKER, S.C. 2003. Synonomy of Boophilus Curtice, 1981 with Rhipicephalus Koch, 1844 (Acari: Ixodidae). Systematic Parasitology, 56:169-172.

NORVAL, R.A.I. 1983. The ticks of Zimbabwe. VII. The genus Amblyomma. Zimbabwe Veterinary Journal, 14:292-305.

NORVAL, R.A.I. 1984. Resistance to Boophilus decoloratus in the African Buffalo (Syncerus caffer). Zimbabwe Veterinary Journal, 15:34-35.

RECHAV, Y., ZEEDERBERG, M.E. \& ZELLER, D.A. 1987. Dynamics of African tick (Acari: Ixodoidea) populations in a natural Crimean-Congo hemorrhagic fever focus. Journal of Medical Entomology, 24:575-583.

SKINNER, J.D. \& SMITHERS, R.H.N. 1990. The mammals of the Southern African Subregion. Pretoria: University of Pretoria.

THEILER, GERTRUD 1956. Zoological Survey of the Union of South Africa. Tick Survey. Part IX. The distribution of the three South African Hyalommas or bontpoots. Onderstepoort Journal of Veterinary Research, 27:239-269.

THEILER, GERTRUD 1962. The Ixodoidea parasites of vertebrates in Africa south of the Sahara (Ethiopian region). 
Project S 9958. Report to the Director of Veterinary Services, Onderstepoort. Mimeographed.

VAN DER MERWE, J.H. 1983. National atlas of South West Africa (Namibia). Goodwood Cape: National Book Printers.

VAN DYK, P.J. \& MCKENZIE, A.A. 1992. An evaluation of the effectivity of the scrub technique in quantitative ectoparasite ecology. Journal of Experimental and Applied Acarology, 15:271-283.

WALKER, JANE B. 1974. The ixodid ticks of Kenya. A review of present knowledge of their hosts and distribution. London and Reading: Commonwealth Institute of Entomology.

WALKER, JANE B. \& OLWAGE, A. 1987. The tick vectors of Cowdria ruminantium (Ixodoidea, Ixodidae, genus Amblyom$\mathrm{ma}$ ) and their distribution. Onderstepoort Journal of Veterinary Research, 54:353-379.
WALKER, JANE B. 1991. A review of the ixodid ticks (Acari, Ixodidae) occurring in southern Africa. Onderstepoort Journal of Veterinary Research, 58:81-105.

WALKER, JANE B., KEIRANS, J.E. \& HORAK, I.G. 2000. The genus Rhipicephalus (Acari, Ixodidae): a guide to the brown ticks of the world. Cambridge: Academic Press.

WHITE, F. 1983. The vegetation of Africa. A descriptive memoir to accompany the UNESCO/AETFAT/UNSO vegetation map of Africa. Paris: UNESCO.

YEOMAN, G.H. \& WALKER, JANE B. 1967. The ixodid ticks of Tanzania. A study of the zoogeoraphy of the Ixodidae of an east African country. London and Reading: Commonwealth Institute of Entomology.

ZIEGER, U., HORAK, I.G., CAULDWELL, A.E. \& UYS, A.C. 1998. Ixodid tick infestations of wild birds and mammals on a game ranch in Central Province, Zambia. Onderstepoort Journal of Veterinary Research, 65:113-124. 